\title{
Recent advances in the temporal and spatiotemporal dynamics induced by bromate-sulfite-based $\mathrm{pH}$-oscillators
}

\author{
István Szalai ${ }^{1}$ D · Brigitta Dúzs ${ }^{1}$ • István Molnár ${ }^{1} \cdot$ Krisztina Kurin-Csörgei $^{1}$ • \\ Miklós Orbán ${ }^{1}$
}

Received: 9 November 2021 / Accepted: 1 December 2021 / Published online: 11 December 2021

(c) The Author(s) 2021

\begin{abstract}
The bromate-sulfite reaction-based $\mathrm{pH}$-oscillators represent one of the most useful subgroup among the chemical oscillators. They provide strong $\mathrm{H}^{+}$-pulses which can generate temporal oscillations in other systems coupled to them and they show wide variety of spatiotemporal dynamics when they are carried out in different gel reactors. Some examples are discussed. When $\mathrm{pH}$-dependent chemical and physical processes are linked to a bromate-sulfite-based oscillator, rhythmic changes can appear in the concentration of some cations and anions, in the distribution of the species in a $\mathrm{pH}$-sensitive stepwise complex formation, in the oxidation number of the central cation in a chelate complex, in the volume or the desorption-adsorption ability of a piece of gel. These reactions are quite suitable for generating spatiotemporal patterns in open reactors. Many reaction-diffusion phenomena, moving and stationary patterns, have been recently observed experimentally using different reactor configurations, which allow exploring the effect of different initial and boundary conditions. Here, we summarize the most relevant aspects of these experimental and numerical studies on bromate-sulfite reaction-based reaction-diffusion systems.
\end{abstract}

Keywords Nonlinear dynamics · Oscillatory reactions · Pattern formation · Reaction-diffusion

\section{Introduction}

The $\mathrm{BrO}_{3}{ }^{-}$and $\mathrm{SO}_{3}{ }^{2-}$ are the most frequently used initial constituents in oscillatory chemical reactions. The $\mathrm{BrO}_{3}{ }^{-}$is a strong oxidant, it is capable of oxidizing many organic and inorganic compounds in oscillatory manner. When the $\mathrm{BrO}_{3}{ }^{-}$reacts with the strong reductant $\mathrm{SO}_{3}{ }^{2-}$ in a CSTR, we can observe two types of oscillations

István Szalai

istvan.szalai@ttk.elte.hu

1 Laboratory of Nonlinear Chemical Dynamics, Institute of Chemistry, Eötvös University,

Pázmány Péter sétány 1/A, Budapest 1117, Hungary 
depending on the environment in which the reaction takes place. In a strong acid medium $\left(\left[\mathrm{H}_{2} \mathrm{SO}_{4}\right] \sim 1-2 \mathrm{M}\right)$, the instability arises from the chemistry of $\mathrm{BrO}_{3}{ }^{-}$. In the oscillatory cycle the positive feedback is the autocatalytic formation of intermediate $\mathrm{HBrO}_{2}$, the negative feedback species is $\mathrm{Br}^{-}$, which removes $\mathrm{HBrO}_{2}$ in a fast reaction. In some systems, a catalyst is needed for the oscillations to occur. The oscillators, composed of $\mathrm{BrO}_{3}{ }^{-}$, reductant (e.g., $\mathrm{SO}_{3}{ }^{2-}$ [1]), and $\mathrm{H}_{2} \mathrm{SO}_{4}$ (and catalyst) are referred to as Belousov-Zhabotinsky (BZ)-type systems.

When the reaction between excess $\mathrm{BrO}_{3}{ }^{-}$and $\mathrm{SO}_{3}{ }^{2-}$ takes place in an unbuffered solution, we can encounter $\mathrm{H}^{+}$-autocatalysis in batch reactor, bistability between high and low $\mathrm{pH}$-states or large amplitude $\mathrm{pH}$-oscillations $(\Delta \mathrm{pH} \sim 3-4)$ in a CSTR [2]. The overall stoichiometry in the oxidation of $\mathrm{SO}_{3}{ }^{2-}$ to $\mathrm{SO}_{4}{ }^{2-}$ by $\mathrm{BrO}_{3}{ }^{-}$ is identical to that it is in the highly acidic solution, but the source of nonlinearity is rather different, it originates from the sulfur chemistry. In neutral or slightly basic mixture of $\mathrm{BrO}_{3}{ }^{-}$and $\mathrm{SO}_{3}{ }^{2-}$ the reaction starts very slowly but becomes selfaccelerating when the more reactive $\mathrm{HSO}_{3}{ }^{-}$and $\mathrm{H}_{2} \mathrm{SO}_{3}$ intermediates are formed and oxidized to $\mathrm{H}_{2} \mathrm{SO}_{4}$ with increasing rate. To observe oscillations, the original $\mathrm{pH}$ should be restored. This can be achieved by adding a second substrate to the system that reacts with $\mathrm{BrO}_{3}{ }^{-}$in $\mathrm{a} \mathrm{H}^{+}$-consuming process and it is silent at high $\mathrm{pHs}$ but dominant in the acidic state. The two-substrate $\mathrm{pH}$-oscillators in which the oxidant is $\mathrm{BrO}_{3}{ }^{-}$have a composition of $\mathrm{BrO}_{3}{ }^{-}-\mathrm{SO}_{3}{ }^{2-}-\left[\mathrm{Fe}(\mathrm{CN})_{6}\right]^{4-}$ (abbr. BSF) [3] and $\mathrm{BrO}_{3}{ }^{-}-\mathrm{SO}_{3}{ }^{2-}-\mathrm{Mn}$ (II) (abbr. BSM) [4]. Under special condition, the negative feedback can develop in the $\mathrm{BrO}_{3}{ }^{-}-\mathrm{SO}_{3}{ }^{2-}$ system without the presence of a third component. In this situation, a new route-accompanied with $\mathrm{H}^{+}$-consumption-opens in the $\mathrm{BrO}_{3}{ }^{-}$oxidation of $\mathrm{SO}_{3}{ }^{2-}$. It is worth to mention that the highly alkaline $\mathrm{Na}_{2} \mathrm{SO}_{3}$ input solution contributes significantly to the overall negative feedback in each oscillators. The $\mathrm{BrO}_{3}{ }^{-}-\mathrm{SO}_{3}{ }^{2-} \mathrm{pH}$-oscillator (abbr. BS) can be viewed as minimal version in the subgroup of $\mathrm{SO}_{3}{ }^{2-}$-based $\mathrm{pH}$-oscillatory reactions. Its extended versions show $\mathrm{pH}$-oscillations in a wider range of the experimental parameters, and they exhibit richer dynamics than the simplest one. The composite reactions (Eqs. 1-4), the brutto process in the $\mathrm{H}^{+}$-autocatalytic $\mathrm{BrO}_{3}{ }^{-}$oxidation of $\mathrm{SO}_{3}{ }^{2-}$ (Eq. 5) and the negative feedback reactions in the BS (Eq. 8), BSF (Eq. 6) and the BSM (Eqs. 7a and $7 \mathrm{~b}$ ) systems are listed in Table 1.

Table 1 The composite and overall chemical reactions that play essential role in the $\mathrm{pH}$-oscillations observed in the $\mathrm{BS}, \mathrm{BSF}$ and BSM systems

$$
\begin{aligned}
& \mathrm{SO}_{3}^{2-}+\mathrm{H}^{+} \rightleftharpoons \mathrm{HSO}_{3}^{-} \\
& \mathrm{HSO}_{3}^{-}+\mathrm{H}^{+} \rightleftharpoons \mathrm{H}_{2} \mathrm{SO}_{3} \\
& \mathrm{BrO}_{3}^{-}+3 \mathrm{SO}_{3}^{2-}+3 \mathrm{H}^{+} \rightarrow \mathrm{Br}^{-}+3 \mathrm{SO}_{4}^{2-}+3 \mathrm{H}^{+} \\
& \mathrm{BrO}_{3}^{-}+3 \mathrm{HSO}_{3}^{-}+3 \mathrm{H}^{+} \rightarrow \mathrm{Br}^{-}+3 \mathrm{SO}_{4}^{2-}+6 \mathrm{H}^{+} \\
& 2 \mathrm{BrO}_{3}^{-}+3\left(\mathrm{SO}_{3}^{2-}+\mathrm{HSO}_{3}^{-}\right)+6 \mathrm{H}^{+} \rightarrow 2 \mathrm{Br}^{-}+6 \mathrm{SO}_{4}^{2-}+9 \mathrm{H}^{+} \\
& \mathrm{BrO}_{3}^{-}+6\left[\mathrm{Fe}(\mathrm{CN})_{6}\right]^{4-}+6 \mathrm{H}^{+} \rightarrow \mathrm{Br}^{-}+6\left[\mathrm{Fe}(\mathrm{CN})_{6}\right]^{3-}+3 \mathrm{H}_{2} \mathrm{O} \\
& \mathrm{BrO}_{3}^{-}+3 \mathrm{Mn}^{2+}+3 \mathrm{H}_{2} \mathrm{O} \rightarrow \mathrm{Br}^{-}+3 \mathrm{MnO}(\mathrm{OH})^{+}+3 \mathrm{H}^{+} \\
& \left.\mathrm{MnO}^{-} \mathrm{OH}\right)^{+}+2 \mathrm{HSO}_{3}^{-}+2 \mathrm{H}^{+} \rightarrow \mathrm{Mn}^{2+}+\mathrm{HS}_{2} \mathrm{O}_{6}^{-}+2 \mathrm{H}_{2} \mathrm{O} \\
& \mathrm{BrO}_{3}^{-}+6 \mathrm{SO}_{3}^{2-}+3 \mathrm{H}^{+} \rightarrow \mathrm{Br}^{-}+3 \mathrm{HS}_{2} \mathrm{O}_{6}^{-}+3 \mathrm{H}_{2} \mathrm{O}
\end{aligned}
$$


In this report, we demonstrate that some $\mathrm{pH}$-oscillators, especially the BS, BSF, and BSM systems can provide strong enough $\mathrm{H}^{+}$-pulses to stimulate repetitive responses in such combined systems where $\mathrm{pH}$-sensitive chemical or physical equilibria are linked to the $\mathrm{pH}$-oscillator. Induced oscillations are generated in the coupled subsystem if the primary oscillator is able to alter the equilibrium between both fore and back directions periodically. In this case the concentration of all components of the equilibrium are forced to oscillate with the frequency of the core oscillator. Bearing this approach in mind, the number and variety of the species that can be incorporated in oscillatory process can significantly be extended.

\section{Induced oscillations in the BS, BSF and BSM systems}

The BS pH-oscillator and its variants are perfectly suitable to play the role of the primary oscillator in the combined systems. They exhibit the largest $\mathrm{pH}$-changes, their operation is practically resistant to the presence of the components of the coupled subsystem, the time the oscillator spends in the high and low $\mathrm{pH}$-states can be tuned to the desired range by varying the experimental parameters. Fig. 1 shows the $\mathrm{pH}$-oscillations in time recorded in the BSM CSTR system.

The simplest manifestation of the induced oscillations are the periodic color changes of an acid-base indicator in a pH-oscillatory mixture. To see more complex induced oscillations, the indicator should be replaced with an appropriate

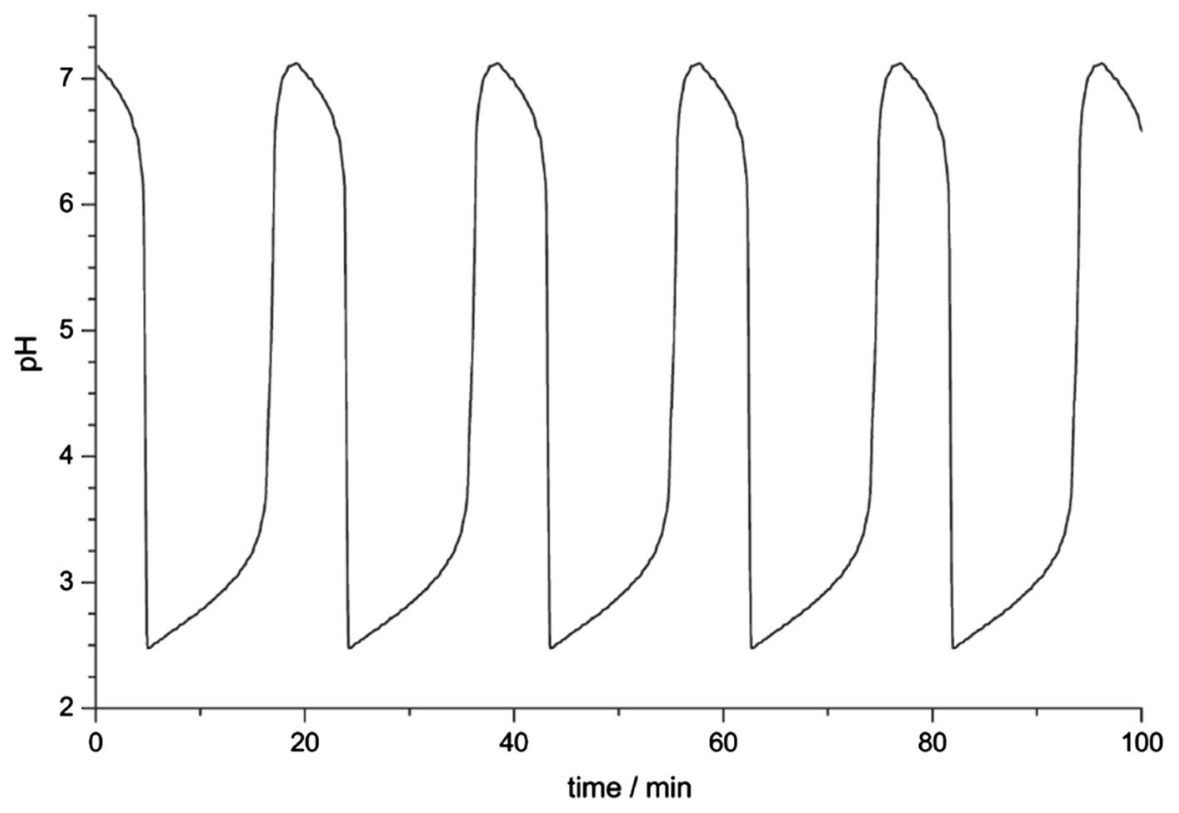

Fig. $1 \mathrm{pH}$-oscillations recorded in the BSM CSTR system. The initial concentrations are $\left[\mathrm{BrO}_{3}{ }^{-}\right]_{0}=$ $0.15 \mathrm{M},\left[\mathrm{SO}_{3}{ }^{2-}\right]_{0}=0.19 \mathrm{M},\left[\mathrm{H}^{+}\right]_{0}=1.2 \times 10^{-2} \mathrm{M},[\mathrm{Mn}(\mathrm{II})]_{0}=2.3 \times 10^{-3} \mathrm{M}$, the reciprocal residence time in the CSTR is $k_{0}=3.7 \times 10^{-3} \mathrm{~s}^{-1}$, the temperature is $T=45^{\circ} \mathrm{C}$ 
pH-dependent complexation or precipitation reaction [5]. Here we list interesting examples. It was possible to bring about rhythmic changes in the concentration of some non-redox ions. Periodic $\mathrm{Ca}^{2+}$ pulses were generated in the BSF-CaEDTA flow system [6]. The BSF oscillator does not react with CaEDTA, however this complex undergoes dissociation at low $\mathrm{pHs}$ but stable in high $\mathrm{pHs}$, which results in two orders of magnitude changes in the concentration of the free $\mathrm{Ca}^{2+}$ during an oscillatory cycle. Oscillations in the free $\left[\mathrm{Al}^{3+}\right]$ were observed in the BSF$\mathrm{Al}\left(\mathrm{NO}_{3}\right)_{3}$ flow system due to the formation of highly insoluble $\mathrm{Al}(\mathrm{OH})_{3}$ above $\mathrm{pH} 4$ and its complete dissolution at $\mathrm{pH}<4$. Periodic pulses in the $\left[\mathrm{F}^{-}\right]$were measured in the $\mathrm{BSM}-\mathrm{Al}\left(\mathrm{NO}_{3}\right)_{3}-\mathrm{NaF}$ combined system where two equilibria, a precipitation and a complex formation, were coupled to each other and to the BSF oscillator [7]. In this case, at low pHs, the $\left[\mathrm{F}^{-}\right]$is low as it is bound in the complex $\left(\mathrm{Al}^{3+}+4 \mathrm{~F}^{-} \rightarrow \mathrm{AlF}_{4}^{-}\right)$, at high pHs, the free $\left[\mathrm{F}^{-}\right]$is high due to the formation of highly insoluble $\mathrm{Al}(\mathrm{OH})_{3}$ (in reaction $\mathrm{AlF}_{4}{ }^{-}+3 \mathrm{OH}^{-} \rightarrow \mathrm{Al}(\mathrm{OH})_{3}+4 \mathrm{~F}^{-}$), which drives out the $\mathrm{F}^{-}$from the complex.

The other way of producing periodic ion pulses is based on the observation that in the $\mathrm{BrO}_{3}{ }^{-}-\mathrm{SO}_{3}{ }^{2-}$ flow system the actual $\left[\mathrm{SO}_{3}{ }^{2-}\right]$ is close to zero at the minimum $\mathrm{pHs}$, but it is maximum at high $\mathrm{pHs}$. Some divalent metal ions, namely $\mathrm{Ca}^{2+}, \mathrm{Cd}^{2+}, \mathrm{Zn}^{2+}, \mathrm{Ni}^{2+}$ and $\mathrm{Co}^{2+}\left(\right.$ abbr. $\left.{ }^{2+}\right)$ form precipitate with $\mathrm{SO}_{3}{ }^{2-}\left(\mathrm{MSO}_{3}\right.$, $K_{\mathrm{s}} \sim 10^{-8}-10^{-7}$ ). When solutions of $\mathrm{BS}$ and $\mathrm{M}^{2+}$ are introduced into a flow reactor, the mixture is rather cloudy at the high $\mathrm{pH}$-state, but it is crystal clear at the acidic state where all $\mathrm{SO}_{3}{ }^{2-}$, including that bound in the precipitate is oxidized by $\mathrm{BrO}_{3}{ }^{-}$to soluble $\mathrm{SO}_{4}{ }^{2-}$. Beside visual observation of the periodic appearance and disappearance of the free $\mathrm{M}^{2+}$, if $\mathrm{M}^{2+}=\mathrm{Ca}^{2+}$ or $\mathrm{Cd}^{2+}$ their concentration can be followed by ion selective electrode as well [8]. In this example, the oxidant $\mathrm{BrO}_{3}{ }^{-}$and the reductant $\mathrm{SO}_{3}{ }^{2-}$ participate directly in bringing about induced oscillations.

Even more complicated forced periodical phenomenon appeared when the BS oscillator was coupled to the highly $\mathrm{pH}$-dependent stepwise complex formation reaction between $\mathrm{Ni}^{2+}$ and histidine (abbr. His) [9]. In this case, periodically changing species distribution in the aqueous $\left[\mathrm{Ni}^{2+}\right]$, the $\left[\mathrm{NiHis}^{+}\right]$and the $\left[\mathrm{Ni}(\mathrm{His})_{2}\right]$ were observed and recorded by simultaneous measurement of the $\mathrm{pH}$ and the light absorbances at the characteristic wavelengths of these species.

Periodic changes in the oxidation number of some redox ions may be generated if the ion in question is involved in the oscillatory mechanism, as Ce(IV) and $\mathrm{Mn}$ (II) do in the BZ-reaction. Similar dynamical phenomenon was found to occur in the BS system coupled to complex formation reaction between $\mathrm{Co}^{2+}$ and histidine [10]. During the $\mathrm{pH}$-oscillatory cycle, no reactions take place at $\mathrm{pH}<4$ but above this value the $\mathrm{Co}$ (II) undergoes $\mathrm{SO}_{3}{ }^{2-}$-induced air oxidation to $\mathrm{Co}(\mathrm{III})$, which is stabilized in the highly inert $\mathrm{Co}(\mathrm{III})$-histidine chelate. The original composition is restored at the acidic pHs when the $\mathrm{Co}$ (III) complex is washed out from the reactor and the reagents are replenished by the continuous flow.

There are several examples of using BS systems to induce oscillations in physical properties of coupled subsystem. When a piece of poly(acrylic acid) cryogel was suspended in the BSF pH-oscillatory mixture, threefold changes in the volume of the gel were observed. The transitions occurred fast, the repetitive shrinking and swelling did not destroy the gel structure. These features make this system suitable 
to be used as a dynamical unit in molecular motors [11]. Reversible adsorption-desorption of $\mathrm{Ag}$ nanoparticles (obtained by reduction of $\mathrm{AgNO}_{3}$ ) was detected on the surface of a honeycomb-patterned $\mathrm{pH}$-sensitive hydrogel when a film of this gel was fixed in the BSF batch-like $\mathrm{pH}$-oscillator. The phenomenon was supposed to arise from the formation of different morphology on the surface of the gel at low and high $\mathrm{pH}[12]$.

\section{Reaction-diffusion patterns in one-side-fed gel reactors}

The spatiotemporal dynamics of the activatory-inhibitory reactions based on the $\mathrm{IO}_{3}{ }^{-}-\mathrm{SO}_{3}{ }^{2-}$ and the $\mathrm{H}_{2} \mathrm{O}_{2}-\mathrm{SO}_{3}{ }^{2-}$ reactions have been thoroughly studied after clarifying the origin of the patterns in the $\mathrm{IO}_{3}{ }^{-}-\mathrm{SO}_{3}{ }^{2-}-\left[\mathrm{Fe}(\mathrm{CN})_{6}\right]^{4-}$ (FIS) system $[13,14]$. The Swinney group has observed a large variety of FIS patterns in the early' $90 \mathrm{~s}[15,16]$. However, the explanation of the origin of the differential diffusion that leads to the formation of the stationary patterns was missing. It turned out that the presence of low mobility weak acid functions plays a crucial role as they can induce an effective slowing down of the $\mathrm{H}^{+}$ions by immobilizing these species through fast reversible protonation of these functions [13, 14]. Based on this finding, the pattern forming capacity of several similar systems has been successfully explored. Turing and other stationary and dynamic patterns have been found and studied experimentally in the $\mathrm{IO}_{3}{ }^{-}-\mathrm{SO}_{3}{ }^{2-}$-thiourea [17], $\mathrm{IO}_{3}{ }^{-}-\mathrm{SO}_{3}{ }^{2-}-\mathrm{S}_{2} \mathrm{O}_{3}{ }^{2-}$ [18], $\mathrm{H}_{2} \mathrm{O}_{2}-\mathrm{SO}_{3}{ }^{2-}-\left[\mathrm{Fe}(\mathrm{CN})_{6}\right]^{4-}$, and in the $\mathrm{H}_{2} \mathrm{O}_{2}-\mathrm{SO}_{3}{ }^{2-}-\mathrm{HCO}_{3}{ }^{-}$systems $[19,20]$. These experiments were made in open one-side-fed gel reactors (OSFR), where a piece of hydrogel, typically agarose, is in contact through a single surface with the content of a CSTR. The sketch of an OSFR is presented in Fig. 2a. Different reactors have been designed to follow the 3D patterns along the direction of the diffusive feed (Fig. 2b) and perpendicular to that (Fig. 2c).
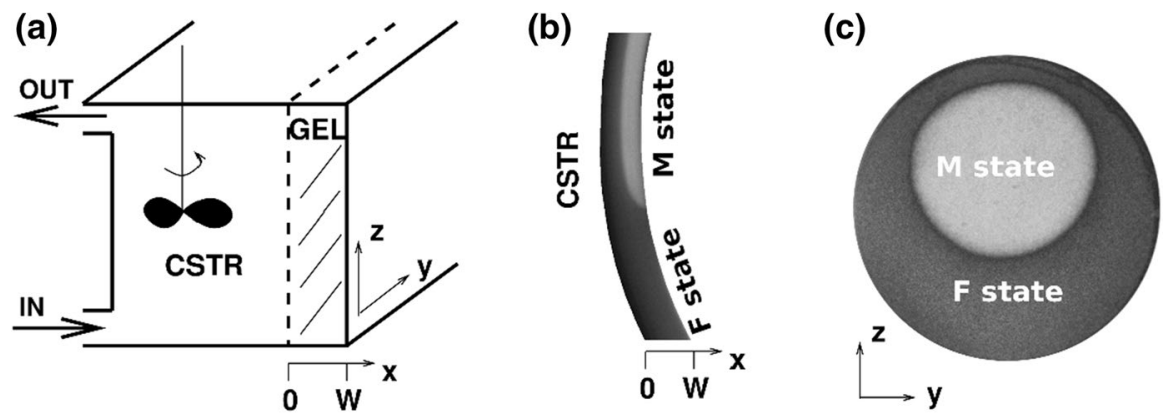

Fig. 2 The basic configuration of one-side-fed gel reactors to study spatiotemporal reaction-diffusion structures (a), and different views of experimentally observed propagating fronts (b and $\mathbf{c}$ ) in the BSF reaction. The CSTR sets a Dirichlet boundary condition at the CSTR/gel surface (dashed line in panel a). The $\mathrm{pH}$-change is monitored by bromocresol green indicator $\left(\mathrm{p} K_{\mathrm{a}}=4.8\right)$. In the pictures (panels $\mathbf{b}$ and $\mathbf{c}$ ) at the dark regions, the $\mathrm{pH}$ is above 6 , while at the light regions, it is below 4 . For details see Reference [23] 
Study of the spatiotemporal dynamics of the core BS reaction-diffusion (RD) system in an OSFR resulted in observation of spatial bistability between two different spatial states and spatiotemporal oscillations [21]. When the gel is fed from the CSTR with a mixture of the reactants, at high $\mathrm{pH}$ and low extent of reaction the gel content can be in a quasi-homogeneous (high $\mathrm{pH}$ ) $\mathrm{F}$ (flow) state or in a mixed $\mathrm{M}$ state characterized by a color switch of the $\mathrm{pH}$-indicator that settles parallel to the CSTR/gel interface. These stationary spatial sates can also be observed in the extended versions of BS system, as it is illustrated in Fig. $2 b$ and c. The stability domain of these spatial states may overlap, which defines spatial bistability. The appearance of spatiotemporal oscillations in the core BS system is originated from the kinetics of the reaction. It is known that the partial oxidation of $\mathrm{SO}_{3}{ }^{2-}$ to dithionate ions (Eq. 8) creates a $\mathrm{H}^{+}$-consuming pathway, which provides an effective negative feedback. Numerical simulations have revealed this explanation [21].

Recently, we have studied how the introduction of another $\mathrm{H}^{+}$-consuming reaction, which is independent of the oxidation of $\mathrm{SO}_{3}{ }^{2-}$ ions, enriches the $\mathrm{BS}$ reaction dynamics [22, 23]. We have explored experimentally and numerically the behavior of the BSF reaction and its coupling to the equilibrium reaction between $\mathrm{Ca}^{2+}$ and EDTA [24].

The primary negative feedback process in the BSF system is the reaction between $\mathrm{BrO}_{3}{ }^{-}$and $\left[\mathrm{Fe}(\mathrm{CN})_{6}\right]^{4-}$. The BSF RD system shows spatial bistability between the $\mathrm{F}$ and $\mathrm{M}$ states (Fig. $2 \mathrm{~b}$ and $\mathrm{c}$ ) at low input feed concentration of $\left[\mathrm{Fe}(\mathrm{CN})_{6}\right]^{4-}$. An appropriate perturbation of the system in the domain of spatial bistability induces a moving front. However, above a critical input feed concentration of $\left[\mathrm{Fe}(\mathrm{CN})_{6}\right]^{4-}$, spatiotemporal oscillations, $\mathrm{pH}$ waves can develop [22]. During this phenomenon, first an acidic $M$ state-like region appears, which expands. Due to the negative feedback process, the middle part of this $\mathrm{M}$ state-like region recovers to the original high $\mathrm{pH}$-state after some delay. At this point the cycle can restart. Snapshots of the
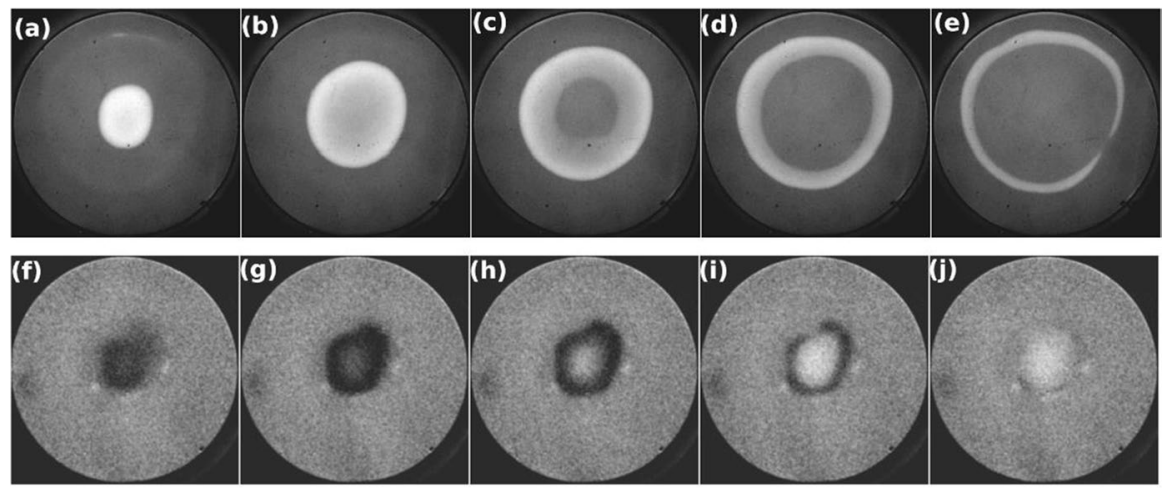

Fig. 3 Snapshots of sustained spatiotemporal $\mathrm{pH}$-waves in the BSF system $(\mathbf{a}-\mathbf{e})$ and $\mathrm{Ca}^{2+}$ waves in the coupled BSF-CaEDTA system $(\mathbf{f}-\mathbf{j})$. The period time of the spatiotemporal oscillations is about $20 \mathrm{~min}$. The $\mathrm{pH}$ and $\mathrm{Ca}^{2+}$ concentration changes are monitored by bromocresol green and arsenazo III indicator, respectively. In snapshots a-e in the dark regions, the $\mathrm{pH}$ is above 6 , while in the light regions, it is below 4. In snapshots $\mathbf{f}-\mathbf{j}$ at the dark regions, the $\mathrm{Ca}^{2+}$ concentration is high. For details see References [22] and [24] 
corresponding experiments are shown in Fig. 3a-e. Numerical simulations successfully reproduced the experimental observations [23].

The robust formation of $\mathrm{pH}$-patterns with a relatively large $\mathrm{pH}$ step makes the $\mathrm{BSF}$ reaction a perfect candidate to create chemically coupled RD systems. In this case, the spatiotemporal $\mathrm{pH}$-variation is used to drive a $\mathrm{pH}$-sensitive equilibrium reaction, the complexation of $\mathrm{Ca}^{2+}$ ions by EDTA [24]. The calcium patterns are visualized by using arsenazo III indicator (Fig. $3 \mathrm{f}-\mathrm{j}$ ). The dynamics of this coupled system seem to be very sensitive to small changes in the thickness of the gel, which results in the localization of the waves. Besides the wave phenomena, stabilization of spots has also been observed [24]. This behavior indicates that the feedback of the driven CaEDTA equilibrium on the driving BSF RD system cannot be neglected. Differential diffusion may result in slowing down the effective diffusivity of $\mathrm{H}^{+}$ions by the interaction of the protonated CaEDTA with the agarose matrix.

In RD systems, stationary patterns can form in the presence of long-range inhibition, e.g., slow diffusion of the activator compared to that of the inhibitor. Sodium polyacrylate (NaPAA), a large molecular weight molecule, has been used to ensure long-range inhibition in the BSF reaction. The different observed pattern formation scenarios are shown in Fig. 4.

A labyrinthine pattern may form when the $M$ state becomes unstable. The repulsive interaction between the remaining high $\mathrm{pH}$-islands avoids their fusion, and finally a labyrinthine pattern settles (Fig. 4a and e). Similar patterns also form by the instability of the F state (Fig. $4 \mathrm{~b}$ and $\mathrm{f}$ ), when at the beginning $\mathrm{M}$ state islands and filaments appear. At higher input feed concentration of $\left[\mathrm{Fe}(\mathrm{CN})_{6}\right]^{4-}$ ions, spatiotemporal oscillations develop from the $\mathrm{F}$ state. The waves create stationary filaments or spots when they approach another existing stationary stripe. The oscillatory region shrinks and vanishes at the expense of the stationary pattern (Fig. 4c and g). At last, we have observed standard Turing instability: the

(a)

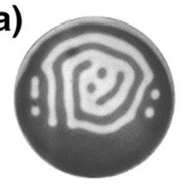

(e)

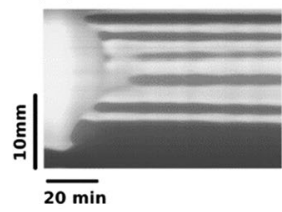

(b)

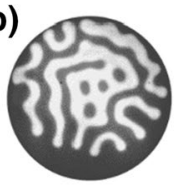

(f)

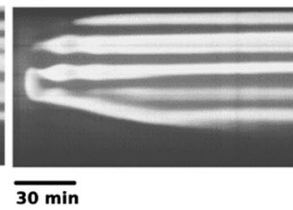

(c)

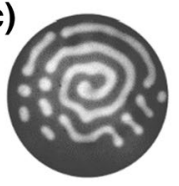

(g)

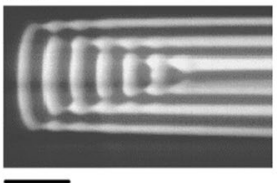

$\overline{20 \mathrm{~min}}$ (d)

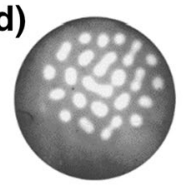

(h)

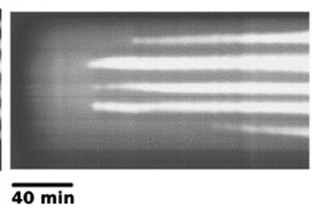

Fig. 4 Stationary pH-patterns in the BSF system in the presence of NaPAA at increasing concentration of $\left[\mathrm{Fe}(\mathrm{CN})_{6}\right]^{4-}$ in an OSFR. The final patterns $(\mathbf{a}-\mathbf{d})$ and the space-time plots $(\mathbf{e}-\mathbf{h})$ present their development. The input feed concentration of the $\left[\mathrm{Fe}(\mathrm{CN})_{6}\right]^{4-}$ is $10 \mathrm{mM}(\mathbf{a}), 15 \mathrm{mM}(\mathbf{b}), 25 \mathrm{mM}(\mathbf{c})$, and $35 \mathrm{mM}$ (d). For details see Reference [23] 
formation of the low $\mathrm{pH}$-spot pattern follows a hexagonal symmetry (Fig. $4 \mathrm{~d}$ and h). The typical wavelength of these patterns is $\lambda=2.7 \mathrm{~mm}$.

\section{Reaction-diffusion patterns in gel reactors with separated sources of reactants}

In an OSFR, the reactions take place both in the CSTR and in the gel. The natural operation method (i.e., the use of an appropriately large flow rate) keeps the CSTR on an "unreacted" steady state, limiting the available parameter space. A simple way to fully avoid the progress of reactions in the feeding reservoirs is the separation of the reactants and the use of different sources for the primary reactants. Different reactors have been designed for this purpose. The two-side-fed gel reactors (TSFR) consist of two open tanks, which are separated by a gel, and the gel is fed from its two opposite sides (Fig. 5a). This configuration creates strong cross-gradients for the reactants. Another way is to soak the two gels with reactants and push them together (Fig. 5b). As the reactants diffuse toward each other, they can react, and transient dynamical phenomena may occur. Recently, a new design has been proposed, where channels are made in a hydrogel (Fig. 5c). The solutions of the reactants flow through the channels and diffuse into the gel. A significant difference between this construction and the TSFR configuration is that here the diffusion field is inhomogeneous. Before our investigations, none of these reactors was used to create and study pattern formation in $\mathrm{H}^{+}$-autocatalytic reactions, like the $\mathrm{BS}$ reaction-based ones.

During the last three years, we have started to explore systematically the RD dynamics of $\mathrm{H}^{+}$-autocatalytic reactions in reactors with separated sources of reactants [25-27]. In our experiments, the BS-based reactions-above all the BSF system-were preferred to use. We separated the reactants of the autocatalytic process (i.e., acid and oxidant) on different sides, while the species responsible for the negative feedback (i.e., $\mathrm{SO}_{3}{ }^{2-}$ and $\left[\mathrm{Fe}(\mathrm{CN})_{6}\right]^{4-}$ in particular cases) were placed symmetrically. The TSFR and the multi-channel reactors are of open configurations, where stationary spatial states can be sustained. These states are indicated by $\mathrm{F}$ and $\mathrm{M}$ (like in an OSFR). In the F state, the whole RD domain is in a low-extent-of-reaction state

(a)

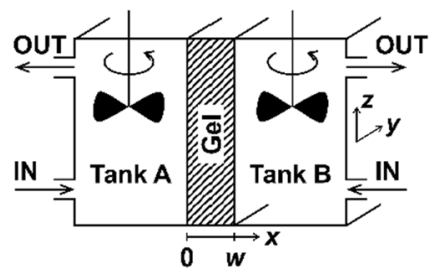

(b)

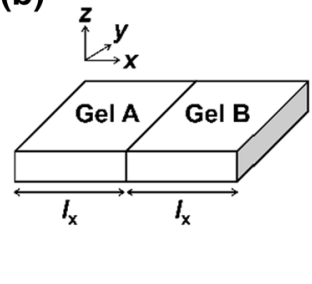

(c)

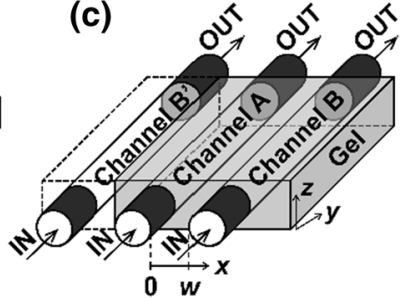

Fig. 5 Gel reactors with separated sources of reactants. Two-side-fed gel reactor (TSFR) with two separated, continuously fed tanks (a). Two-layer closed gel reactor where two initially homogeneously filled gel halves of the same size are connected (b). Cuboid hydrogel device fed from two or three (dashed) embedded flow-through channels (c). A and B indicate different subsets of reactants. For details see References [25-27] 
(a)

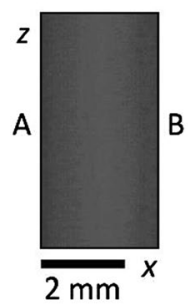

(e)

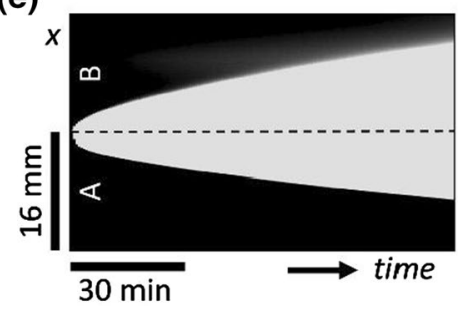

(c)

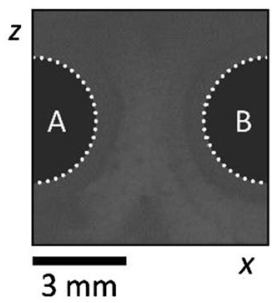

(d)

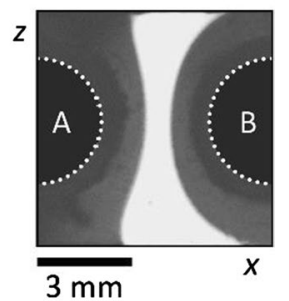

(f)

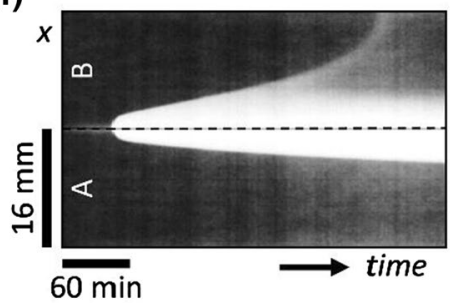

Fig. 6 Stationary spatial states in a two-side fed gel reactor $(\mathbf{a}, \mathbf{b})$ and in the gel reactor with two flowthrough channels $(\mathbf{c}, \mathbf{d})$ by using the BSF reaction. Unstructured F state (a, c) and three-zoned M state (b, d) in the gel. Autocatalytic front propagation in the BS system (e) and localized high-extent-of-reaction zones (f) in the BSF system created in the two-layer gel reactor, represented in space-time plots. A and $\mathrm{B}$ indicate the separation of the acid and the $\mathrm{BrO}_{3}{ }^{-}$reactants, respectively. For details see References [25-27]

(Fig. 6a and c). The M state, in which the gel has a localized high-extent-of-reaction zone, can be reached at higher values of the boundary acid concentration (Fig. 6b and d). Here, contrary to an OSFR (Fig. 2b), the high-extent-of-reaction zone is located between two low-extent-of-reaction zones (Fig. 6b). In the two-channel configuration, the sources of the reactants are framed by the gel, so the RD field is not uniform in direction $z$, and the high-extent-of-reaction zone of the M state widens on the top and bottom (Fig. 6d). The boundary concentrations of the separated species and the gel thickness (or channel distance) in direction $x$ are good control parameters to tune the width and position of the middle zone of the M state of the gel. In the autocatalytic subsystem and at low concentrations of $\left[\mathrm{Fe}(\mathrm{CN})_{6}\right]^{4-}$, spatial bistability was found between the F and $\mathrm{M}$ states in both open geometries. The twolayer gel reactor is a closed configuration; thus, we cannot sustain time-independent stationary states. In the autocatalytic subsystem, a fast autocatalytic front propagates through the gel, starting from the contact zone of the two halves (Fig. 6e). In the presence of $\left[\mathrm{Fe}(\mathrm{CN})_{6}\right]^{4-}$ (negative feedback), localized high-extent-of-reaction zones develop in the contact zone and in a separated region (Fig. 6f) (separation of the two reaction zones begins after $4 \mathrm{~h}$ ). The middle one is quasi-stabilized, but the upper one moves towards the upper edge of the reactor.

After finding spatial bistability, the negative feedback was increased in order to look for more complex dynamic phenomena. In the TSFR and the two-channel gel reactor, we created sustained, localized $\mathrm{pH}$ waves above a critical concentration of 
(a)

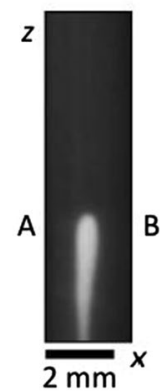

(c)

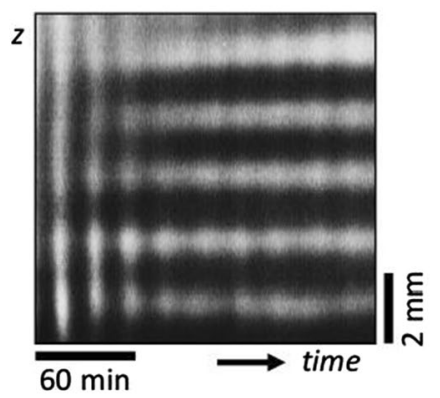

(b)
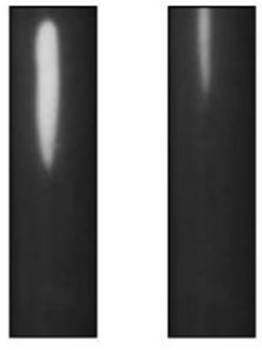

(d)

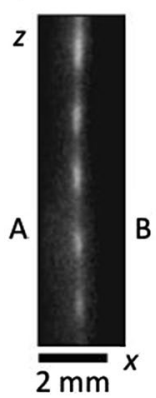

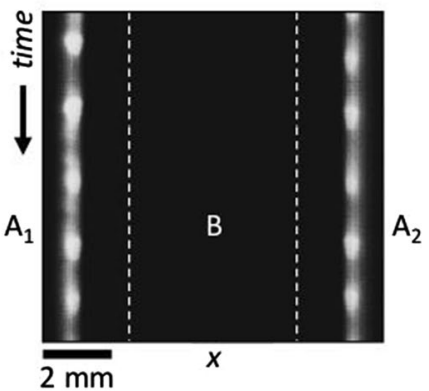

(e)

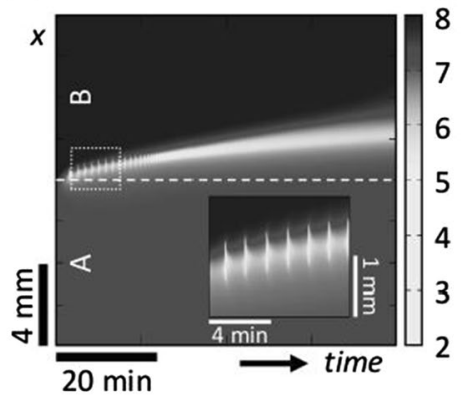

Fig. 7 Spatiotemporal waves and stationary Turing structures with separated sources of reactants in the BSF system. Sustained localized $\mathrm{pH}$-waves in the TSFR on consecutive snapshots with $\Delta t=4 \mathrm{~min}(\mathbf{a})$. Space-time plot of two neighbouring zones of sustained localized $\mathrm{pH}$ waves in the three-channel gel reactor (b). The borders of the middle channel are indicated by dashed lines. Stabilization of a row of Turing spots in the TSFR on a space-time plot (c) and snapshot of the resulting stationary pattern (d). Simulated space-time plot of the spatiotemporal oscillations in the two-layer gel reactor by using the model of two-substrate $\mathrm{pH}$ oscillators $(\mathbf{e})$. A and $\mathrm{B}$ indicate the separation of the acid and the $\mathrm{BrO}_{3}{ }^{-}$reactants, respectively. For details see references [25-27]

$\left[\mathrm{Fe}(\mathrm{CN})_{6}\right]^{4-}$ (Fig. 7a). The waves propagate in the middle of the two-side-fed gel domain with a period of about $15 \mathrm{~min}$. The flow-through gel reactor can be extended with further channels, thus we can create neighbouring RD zones that may diffusively interact. The time-evolution of two oscillatory zones, being fed through one common channel (B) and two distinct outer channels (A1, A2), can be seen in Fig. 7b. In a TSFR, Turing instability was induced by adding NaPAA. Due to the oscillatory destabilization of the $\mathrm{M}$ state, a localized row of stationary, low-pH spots emerged (Fig. 7c and d). Similar Turing pattern developed starting from the F state, by the consecutive appearance of the spots. In a two-layer gel reactor, the existence of a complex spatiotemporal BSF pattern is highly nontrivial, because of the lack of the continuous supply of the reactants. However, simulations made by the Rábai model of $\mathrm{pH}$-oscillators suggested transient spatiotemporal oscillations in this geometry (Fig. 7e). The reason behind this phenomenon is the fact that the relatively large gel halves can act as transitory reagent sources of the initial reactants, providing 
fuel for several oscillatory cycles. Being very sensitive to the kinetic parameters, this behavior was not produced experimentally neither with the BSF, nor with other $\mathrm{pH}$-oscillators. But it should be noted that the two-layer gel reactor was successful in creating patterns with a $\mathrm{ClO}_{2}-\mathrm{I}_{2}-$ malonic acid oscillator [28].

The price of the flexibility gained by using separated sources of the reactants is the presence of sharp cross-gradients. When TSFR was used to study the spatiotemporal dynamics of the $\mathrm{ClO}_{2}{ }^{-}-\mathrm{I}^{-}$-malonic acid (CIMA) reaction in a TSFR, it was recognized that in this feeding mode, different instabilities might develop at different positions in the gel. This property made difficult the interpretation of the observed patterns [29]. It is essential to recognize that the CIMA reaction has much more complex kinetics than the BS-based reactions. The capability of the CIMA reaction to show temporal oscillations in a batch reactor demonstrates it. The relative simplicity of the BS-based reactions, especially the fact that one of the reactants is nearly totally consumed in the reaction zone, avoids this problem. Patterns develop only in a well-defined region of the gel as gradients naturally localize them. The interaction of the gradients and the nonlinear kinetics is a paradigm of developmental biology since the seminal work of Wolpert [30]. This perspective also makes it challenging to explore the dynamics of $\mathrm{H}^{+}$-self-activated reactions in these reactors.

\section{Conclusions}

Information was collected and experiments were carried out to establish the usability of the bromate-sulfite-based $\mathrm{pH}$-oscillators for producing novel temporal and spatiotemporal dynamical phenomena. From these knowledges and results it was concluded that both BS system and its variants are good-may be the best—candidates among all $\mathrm{pH}$-oscillators for generating rhythmic changes in the concentration of the species or in the states of such a $\mathrm{pH}$-dependent equilibrium reaction that is coupled to the $\mathrm{pH}$-oscillator through $\mathrm{H}^{+}$. Many examples are presented where a BS based primary oscillator forces the linked equilibrium to respond periodically with the frequency of the core system. This kind of induced oscillations may represent a way as oscillations occur in living organisms. The BS based $\mathrm{pH}$-oscillators were successfully used to produce spatial dynamical phenomena as well. So far spatial bistability and Turing structure were observed in a TSFR. The use of other kind of gel reactors, like two-layer closed gel reactor or the recently designed device which is fed from two or three flow-through channels provides best chances to bring about more sophisticated spatial structures.

Acknowledgements This work was supported by the National Research, Development and Innovation Office of Hungary (K134687).

Funding Open access funding provided by Eötvös Loránd University.

Open Access This article is licensed under a Creative Commons Attribution 4.0 International License, which permits use, sharing, adaptation, distribution and reproduction in any medium or format, as long as you give appropriate credit to the original author(s) and the source, provide a link to the Creative Commons licence, and indicate if changes were made. The images or other third party material in this article are included in the article's Creative Commons licence, unless indicated otherwise in a credit line to the 
material. If material is not included in the article's Creative Commons licence and your intended use is not permitted by statutory regulation or exceeds the permitted use, you will need to obtain permission directly from the copyright holder. To view a copy of this licence, visit http://creativecommons.org/licen ses/by/4.0/.

\section{References}

1. Alamgir M, Orbán M, Epstein IR (1983) Inorganic bromate oscillators: bromate-manganous-reductant. J Phys Chem 87:3725-3728

2. Szántó TG, Gy R (2005) pH-Oscillations in the $\mathrm{BrO}_{3}{ }^{-}-\mathrm{SO}_{3}{ }^{2-} / \mathrm{HSO}_{3}{ }^{-}$reaction in a CSTR. J Phys Chem A 109:5398-5402

3. Edblom EC, Luo Y, Orbán M, Kustin K, Epstein IR (1989) Kinetics and mechanism of the oscillatory bromate-sulfite-ferrocyanide reaction. J Phys Chem 93:2722-2727

4. Okazaki N, Gy R, Hanazaki I (1999) Discovery of novel bromate-sulfite pH-oscillators with $\mathrm{Mn}^{2+}$ or $\mathrm{MnO}_{4}{ }^{-}$as a negative feeedbeck species. J Phys Chem 93:10915-10920

5. Kurin-Csörgei K, Epstein IR, Orbán M (2005) Systematic design of chemical oscillators using complexation and precipitation equilibra. Nature 433:139-142

6. Kurin-Csörgei K, Epstein IR, Orbán M (2006) Periodic pulses of calcium ions in a chemical system. J Phys Chem A 110:7588-7592

7. Horváth V, Kurin-Csörgei K, Epstein IR, Orbán M(2008) Oscillations in the concentration of fluoride ions induced by a pH-oscillator. J Phys Chem A 112:4271-4276

8. Horváth V, Kurin-Csörgei K, Epstein IR, Orbán M (2010) Oscillatory concentration pulses of some divalent metal ions induced by a redox oscillator. Phys Chem Chem Phys 12:1248-1252

9. Poros E, Kurin-Csörgei K, Szalai I, Horváth V, Orbán M (2014) Periodic changes in the distribution of species observed in the $\mathrm{Ni}^{2+}$-histidine complex coupled to the $\mathrm{BrO}_{3}{ }^{-}-\mathrm{SO}_{3}{ }^{2-} \mathrm{pH}$-oscillator. J Phys Chem A 118:6749-6756

10. Kurin-Csörgei K, Poros E, Csepiova J, Orbán M (2018) Periodic changes in the oxidation states of the center ionin the cobalt-histidine complex induced by the $\mathrm{BrO}_{3}{ }^{-}-\mathrm{SO}_{3}{ }^{2-} \mathrm{pH}$-oscillator. Chaos 28:053114-053121

11. Bihici C, Karajel S, Demii T, Okay O (2010) Self-oscillating pH-responsing cryogels as possible candidates of soft materials for generating mechanical energy. J Appl Polim Sci 118:2981-2988

12. Jang JH, Orbán M, Wang S, Huh DS (2014) Adsorption-desorption oscillations of nanoparticles on a honeycomb-patterned $\mathrm{pH}$-responsive hydrogel surface in a closed reaction system. Phys Chem Chem Phys 16:25296-25305

13. Szalai I, De Kepper P (2008) Patterns of the ferrocyanide-iodate-sulfite reaction revisited: the role of immobilized carboxylic functions. J Phys Chem A 112:783-786

14. Szalai I, De Kepper P (2008) Pattern formation in the ferrocyanide-iodate-sulfite reaction: the control of space scale separation. Chaos 18:026105

15. Lee KJ, McCormick WD, Pearson JE, Swinney HL (1994) Experimental observation of self-replicating spots in a reaction- diffusion system. Nature 369:215-218

16. Lee KJ, Swinney HL (1995) Lamellar structures and self-replicating spots in a reaction-diffusion system. Phys Rev E 51:1899-1915

17. Horváth J, Szalai I, De Kepper P (2009) An experimental design method leading to chemical Turing patterns. Science 324:772-775

18. Liu H, Pojman JA, Zhao Y, Pan C, Zheng J, Yuan L, Horváth AK, Gao Q (2012) Pattern formation in the iodate-sulfite-thiosulfate reaction-diffusion system. Phys Chem Chem Phys 14:131-137

19. Szalai I, Horváth J, Takács N, De Kepper P (2011) Sustained self-organizing pH- patterns in hydrogen peroxide driven aqueous redox systems. Phys Chem Chem Phys 13:20228-20234

20. Horváth J, Szalai I, De Kepper P (2009) Designing stationary reaction-diffusion patterns in pH selfactivated systems. Acc Chem Res 51:3183-3190

21. Zs V, Szalai I, Boissonade J, De Kepper P (2007) Sustained spatiotemporal patterns in the bromatesulfite reaction. J Phys Chem A 111:8090-8094

22. Molnár I, Szalai I (2015) Pattern formation in the bromate-sulfite-ferrocyanide reaction. J Phys Chem A 119:9954-9961

23. Molnár I, Szalai I (2017) Kinetic and diffusion-driven instabilities in the bromate-sulfite-ferrocyanide system. J Phys Chem A 121:1900-1908 
24. Molnár I, Kurin-Csörgei K, Orbán M, Szalai I (2014) Generation of spatiotemporal calcium patterns by coupling a pH-oscillator to a complexation equilibrium. Chem Commun 50:4158-4160

25. Dúzs B, Szalai I (2018) Design of localized spatiotemporal pH-patterns by means of antagonistic chemical gradients. RSC Adv 8:41756-41761

26. Dúzs B, Szalai I (2018) Front dynamics of pH oscillators with initially separated reactants. React Kinet Mech Cat 123:335-349

27. Dúzs B, Szalai I (2020) A simple hydrogel device with flow-through channels to maintain dissipative non-equilibrium phenomena. Commun Chem 3:1-6

28. Dúzs B, De Kepper P, Szalai I (2019) Turing patterns and waves in closed two-layer gel reactors ACS. Omega 4:3213-3219

29. Dulos E, Davies P, Rudovics B, De Kepper P (1996) From quasi-2D to 3D Turing patterns in ramped systems. Physica D 98:53-66

30. Wolpert L (1969) Positional information and the spatial pattern of cellular differentiation. J Theor Biol $25: 1-47$

Publisher's Note Springer Nature remains neutral with regard to jurisdictional claims in published maps and institutional affiliations. 